\title{
Exemples de sinistres en région parisienne
}

\author{
Accidents examples in Paris area
}

\author{
P. VANDANGEON \\ Directeur de l'Agence Ile-de-France SIMECSOL*
}

Rev. Franç. Géotech. n 58, pp. $7-14$ (janvier 1992)

\section{Résumé}

La sécheresse des années 1989 et 1990 a entraîné un grand nombre de désordres dans les constructions fondées sur des sols argileux. La région parisienne a été particulièrement touchée par ce phénomène dont le présent article décrit le mécanisme. Après avoir analysé les méthodes d'investigation permettant de déterminer, cas par cas, l'origine des désordres, l'auteur indique les solutions à mettre en œuvre pour conforter les bâtiments sinistrés et pour prévenir de tels accidents.

\section{Abstract}

The drought that stroke the country in 1989 and 1990 has been the cause of many damages in buildings founded above clayey soils. The Paris region has been particularly hit by this phenomenon, the mecanism of which is described in this article. After having analysed the investigation methods which allow the determination of the origin of the damage on a case by case basis, the author reviews the relevant methods to comfort the damaged building and prevent such accidents.

\footnotetext{
- 8, avenue Newton, 92350 Le Plessis-Robinson.
} 


\section{ANALYSE PHYSIQUE DU PHÉNOMÈNE}

Du point de vue granulométrique, les argiles sont des sols très fins dont les éléments sont inférieurs à deux microns. Au plan minéralogique, qui ne sera pas développé, il s'agit d'un assemblage d'éléments phulliteux en forme de feuillets, et d'eau.

Les argiles de la région parisienne, présentent à l'état saturé une teneur en eau de l'ordre de 35 à $40 \%$. Compte tenu du fait que le poids spécifique de l'eau est de 2,7 fois inférieur à celui des particules solides, il y a en volume autant d'eau que de solide dans largile. Encore faut-il préciser que les argiles de la région parisienne sont parmi celles dont la teneur en eau est la plus faible. Il n'est pas rare de rencontrer des argiles magnésiennes qui atteignent 70 à $80 \%$ de teneur en eau à la saturation.

Les argiles sont généralement le plancher d'une nappe phréatique et des nappes captives peuvent se mettre en charge sous ces terrains. Le plus souvent, les argiles sont donc en contact avec l'eau par le dessus et parfois par le dessous.

En période de sécheresse, les nappes peuvent arriver à se tarir, si bien que les argiles perdent leur état de saturation par évaporation de l'eau qu'elles contiennent. Ce phénomène est fréquemment accentué par la succion de l'eau au sein de la masse argileuse par des racines d'arbres. Ainsi un peuplier ou un saule adulte, qui a besoin de 3001 d'eau par jour en été, doit-il puiser cette eau dans l'argile elle-même après que la nappe se soit tarie ou qu'il ait contribué à la tarir. Il se développe alors un phénomène de retrait qui se traduit par une diminution de volume de largile et entraîne un tassement (diminution de volume dans le sens vertical) et une fissuration (diminution de volume dans le sens horizontal).

Lorsque le terrain est à nouveau réhydraté, l'eau pénètre dans les fissures et l'argile tend à nouveau vers son volume initial. C'est le phénomène du gonflement.

A ce propos, le terme d'argile gonflante peut être considéré comme un pléonasme car toutes les argiles possèdent la propriété de gonfler et de se rétracter en fonction des variations de teneur en eau. Et il faut bien dire que si les phénomènes de gonflement sont souvent extrêmement spectaculaires, ce sont les phénomènes de retrait qui sont de loin les plus nombreux et qui sont à l'origine de la plupart des sinistres liés à la nature argileuse du terrain de fondation (fig. 1).

\section{TERRAINS DE LA RÉGION PARISIENNE CONCERNÉS. EXEMPLES DE SINISTRE}

Dans la série de la région parisienne, constituée essentiellement de terrains tertiaires (à l'exception des alluvions quaternaires et de la craie secondaire), on compte six couches d'argile qui peuvent être à l'origine de sinistres.

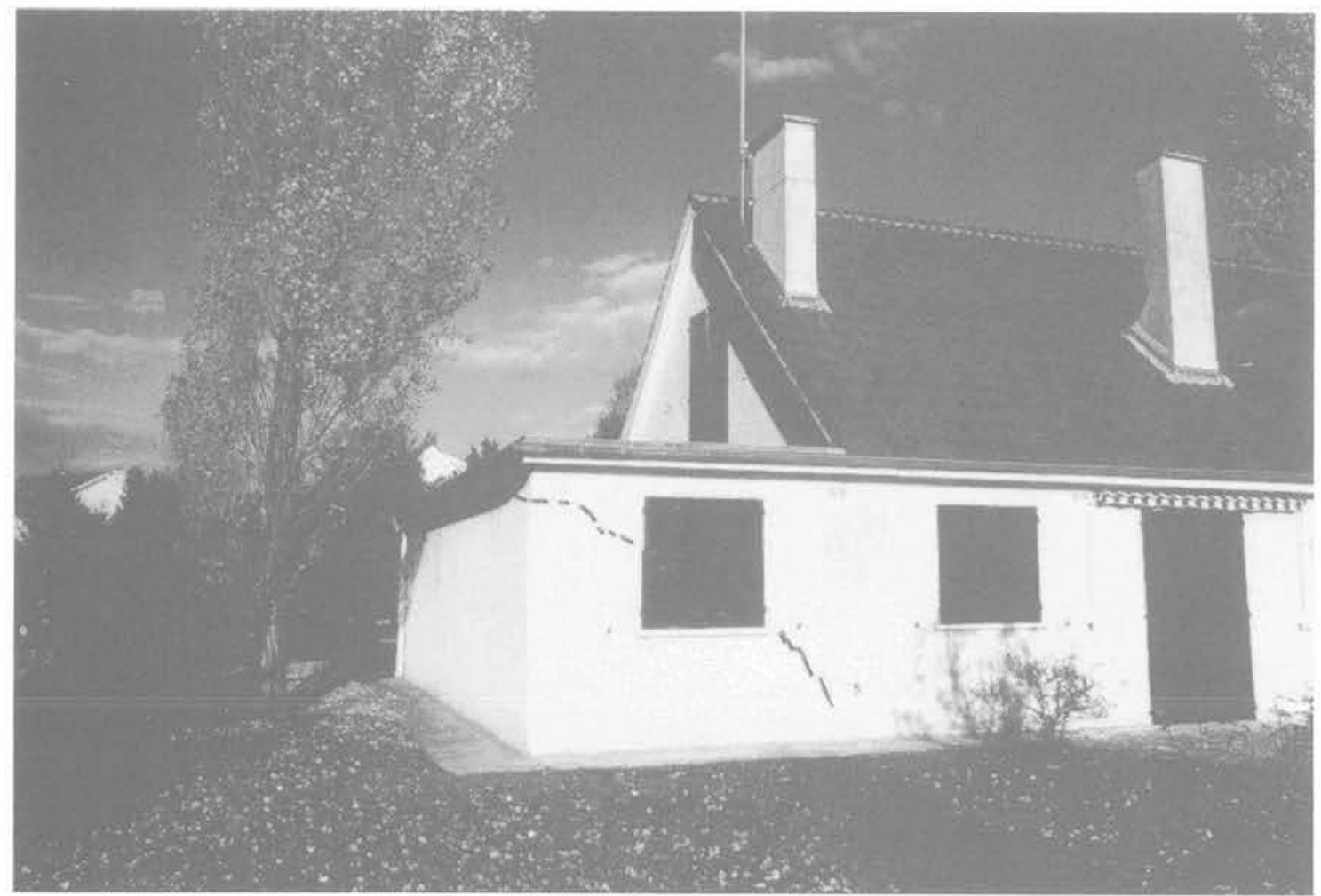

Fig. 1. - (cliché SIMECSOL). Un terrain argileux, une maison individuelle, un peuplier..

les conditions idéales pour une fissure spectaculaire. Le Mesnil-Saint-Denis (Yvelines).

Fig. 1. - Clayey Soil, private house, poplar-tree... best conditions for an enormous crack I Le Mesnil-St-Denis (Yvelines). 
Du haut en bas de la série;

- les argiles à meulière de Beauce (5 $\mathrm{m}$ d'épaisseur moyenne) ;

- les argiles à meulière de Brie $(5 \mathrm{~m}$ d'épaisseur moyenne) ;

- les argiles vertes ( $5 \mathrm{~m}$ d'épaisseur moyenne) qui donnent lieu à la plus grande partie des sinistres parce qu'on les rencontre fréquemment à l'affleurement ;

- les marnes sypragypseuses (et spécialement la partie inférieure correspondant aux marnes d'Argenteuil particulièrement argileuses et qui compte $10 \mathrm{~m}$ d'épaisseur environ) :

- les marnes infragypseuses (5 $\mathrm{m}$ d'épaisseur environ) ;

- les argiles plastiques (10 $\mathrm{m}$ d'épaisseur environ).

En outre, les limons de plateau et les alluvions modernes peuvent donner lieu à des phénomènes, mais plus atténués, de tassement - gonflement.

$\mathrm{Au}$ total, il y a donc $40 \mathrm{~m}$ d'épaisseur de couches d'argile en région parisienne sur une série sédimen- taire d'environ $200 \mathrm{~m}$ de hauteur. C'est dire qu'aucune partie de l'lle-de-France n'est épargnée par le phénomène, d'autant que les terrains argileux déterminant des reliefs mous, ont tendance à fluer dans les pentes et occupent donc d'importantes surfaces (fig. 2).

Mais fort heureusement, ce n'est pas parce que le terrain est argileux qu'il y aura sinistre dû à un phénomène de retrait. Pour qu'il $y$ ait sinistre il faut en effet qu'il y ait un retrait différentiel au droit de la construction considérée et que cette construction ne puisse pas s'adapter à ce mouvement différentiel. Ainsi les immeubles collectifs qui sont généralement plus raides que les maisons individuelles, du moins lorsqu'ils comportent des voiles en béton armé, sont-ils moins vulnérables, si bien que $95 \%$ des sinistres concernent des maisons individuelles. Par ailleurs, la plus grande partie des sinistres ( $80 \%$ environ) se rapportent à des maisons près desquelles se trouvent des saules, des peupliers ou des chênes, ou des végétations denses (fig. 3).

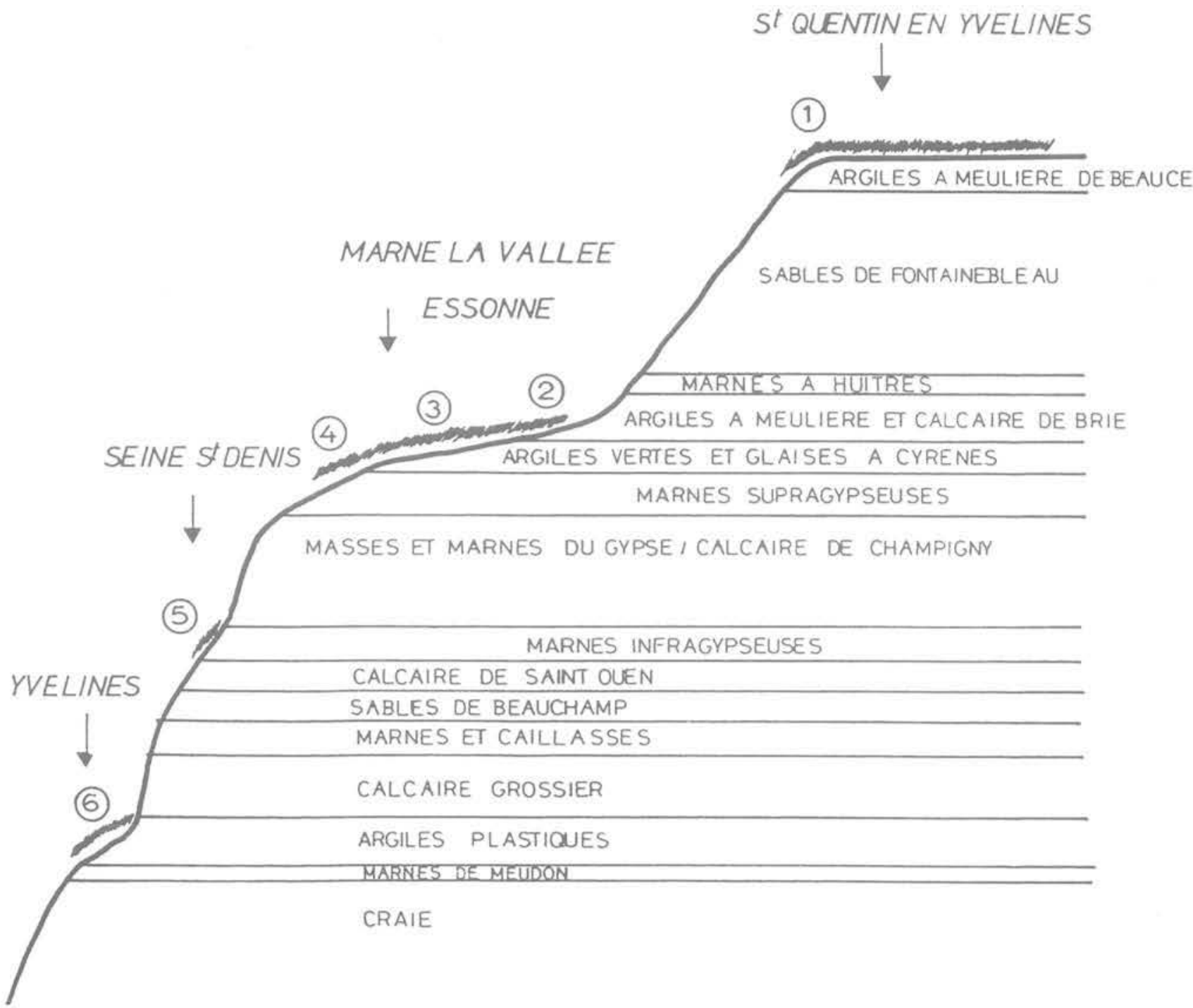

Fig. 2. - Série tertiaire de la région parisienne, - Zones sensibles.

Fig. 2. - Tertiary strastas of Paris region. - Sensitive areas. 


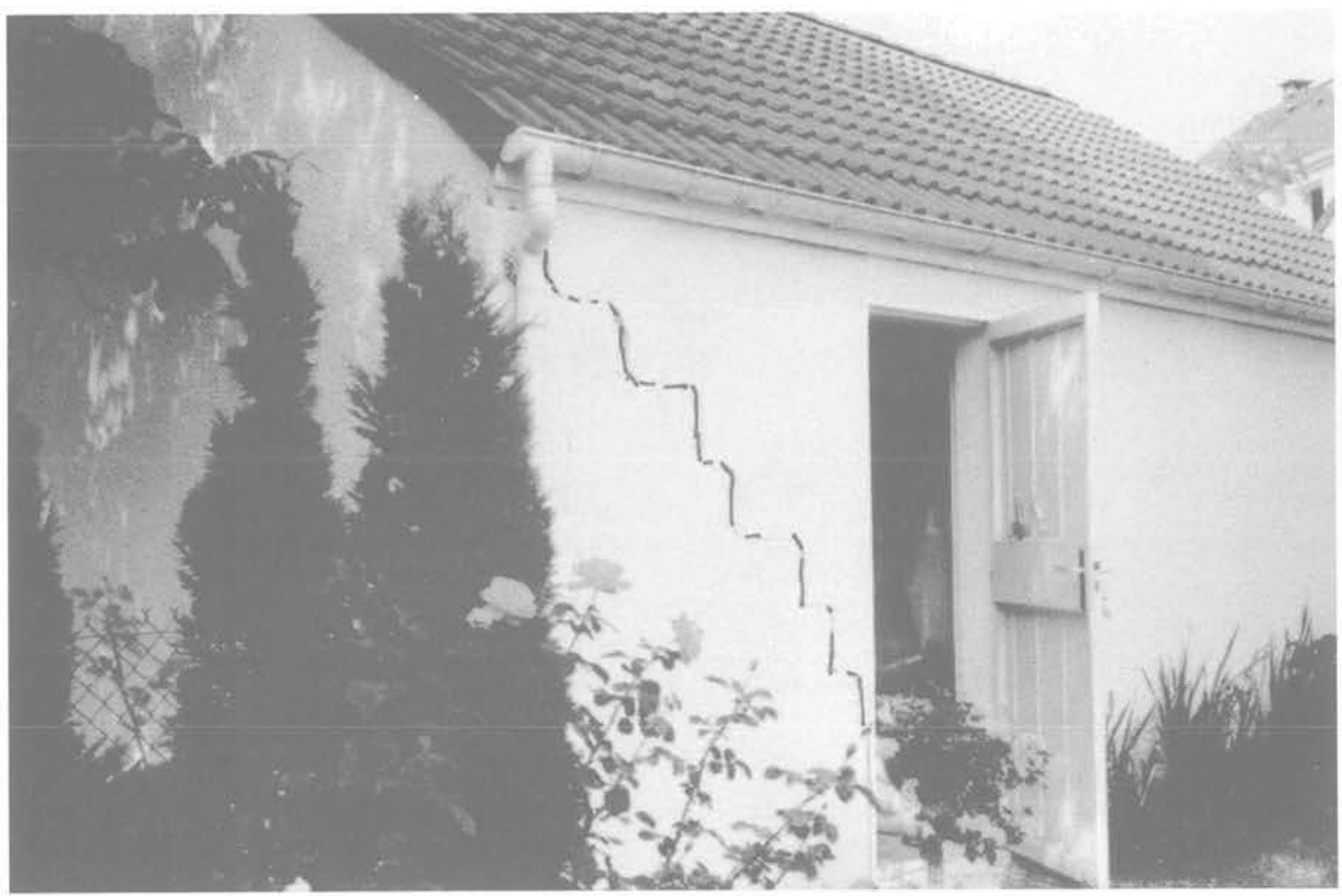

Fig. 3. - (Cliché SIMECSOL). Pavillon fondé sur les marnes infragypseuses à Sarcelles (Seine-St-Denis). Fig. 3. - Villa settled on infra-gypseous marl in Sarcelles (Seine-St-Denis).

Certaines régions sont évidemment plus exposées que d'autres. Ce sont en premier lieu les villes nouvelles de St-Quentin-en-Yvelines et Marne-la-Vallée, la première se situant au droit des argiles à meulière de Beauce et la seconde au droit des argiles vertes et des marnes supragypseuses. Leur vulnérabilité est d'autant plus élevée que lorsque les premières constructions ont été faites, la nappe d'eau se trouvait à faible profondeur, aussi a t-on évité de faire des soussols. Du coup, les fondations étant très superficielles, elles se trouvent directement soumises aux variations de teneur en eau. Par ailleurs, pour agrémenter un paysage souvent ingrat, on a planté des arbres, et quoi de plus vivace que les peupliers et les saules qui aiment l'eau? Avec l'urbanisation croissante, les surfaces ont été imperméabilisées et les nappes se sont lentement taries, si bien que les phénomènes de retrait se sont fortement développés et des sinistres importants se sont produits, bien avant les années sèches de 1989 et 1990 .

D'autres sites sont bien connus pour subir depuis de nombreuses années ce type de phénomène. On peut citer dans le département du Val-de-Marne, les communes de Villecresnes et Roissy-en-Brie où de grands lotissements ont été construits sans précautions particulières avec des fondations très superficielles ou sur des pieux picots ancrés dans l'argile. A l'Ouest de Paris, dans le département des Yvelines, où les argiles plastiques peuvent se trouver à faibe profondeur (Mantes-la-Ville...), de nombreux désordres se sont également produits. Cette liste est loin d'être limitative.

Mais avec la grande sécheresse des années 1989 et 1990, le phénomène s'est étendu à un très grand nombre de constructions pourtant déjà anciennes et dont certaines avaient bien supporté la sécheresse de 1976. Le département de l'Essonne et de la Seineet-Marne ont été ainsi largement touchés ainsi que le sud des Hauts-de-Seine. On a vu des désordres extrêmernent spectaculaires se produire à Paris même, dans le $20^{e}$ arrondissement au-dessus des argiles vertes (fig. 4).

\section{LES FONDATIONS SUR TERRAINS ARGILEUX. HISTORIQUE}

Il est intéressant de faire un retour en arrière sur la manière dont les problèmes ont été abordés par les géotechniciens confrontés aux fondations sur terrains argileux en région parisienne et aux sinistres qui en ont découlés.

Avant 1976, on craignait surtout les phénomènes de gonflement, si bien qu'il était usuel de charger aussi fortement que possible les fondations. On disait alors par exemple que la pression de gonflement des argiles vertes était de $0,2 \mathrm{MPa}$ et que pour éviter des désordres, le taux de travail du sol devait excéder cette valeur, sans toutefois dépasser $0,3 \mathrm{MPa}$ car l'argile reste tout de même compressible...

En fait la pression de gonflement n'est pas une caractéristique intrinsèque d'une argile. Cette pression de gonflement varie en fonction de la teneur en eau. A saturation, une argile ne gonfle pas : par contre un abaissement de sa teneur en eau entraîne un fort potentiel de gonflement. Ainsi une argile plastique 


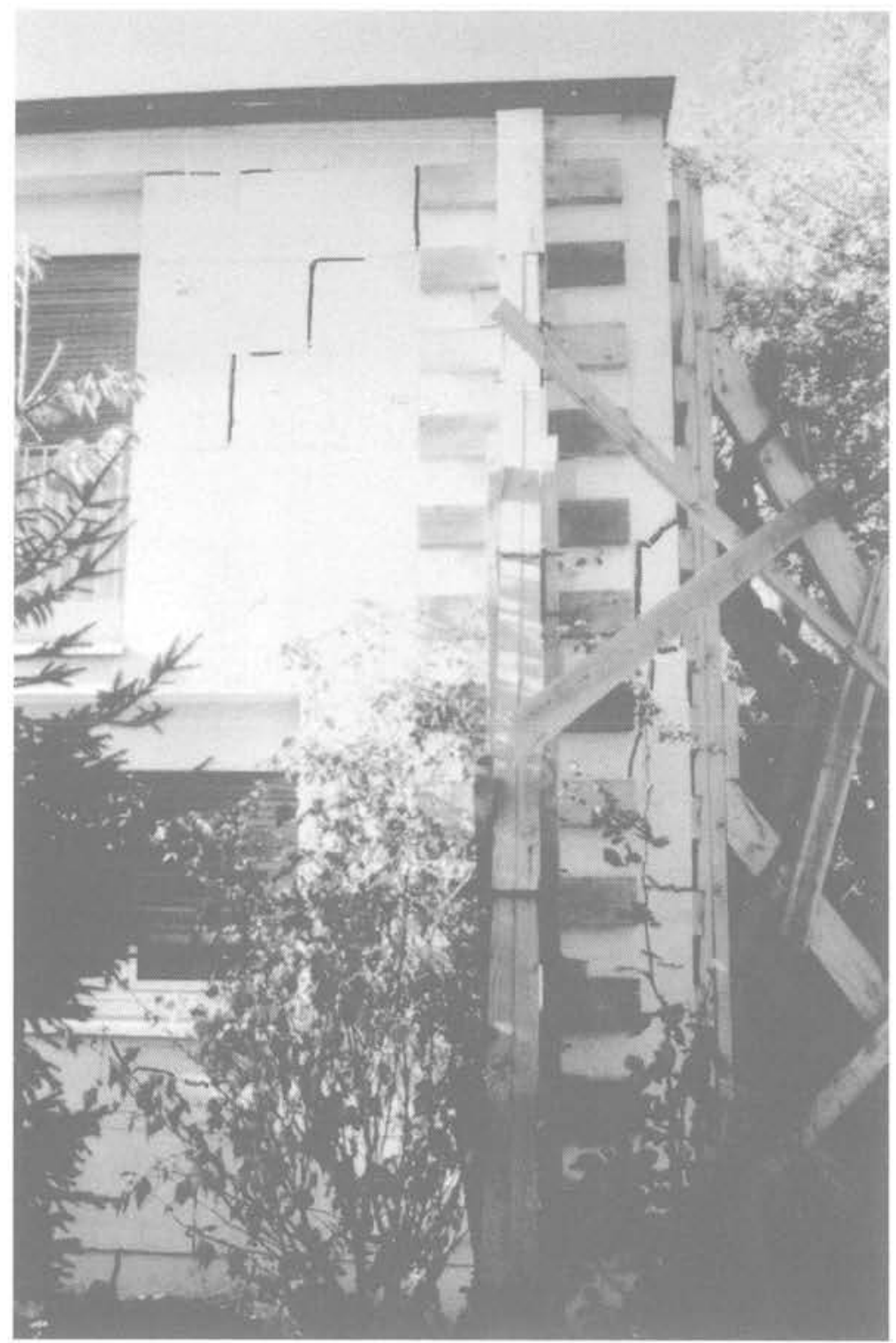

Fig. 4. - (Cliché SIMECSOL). Etaiement d'un pavillon fondé sur les argiles vertes et dont le pignon menaçait de s'écrouler à Quincy-sous-Senart (Seine-et-Marne).

Fig. 4. - Strutting for private house founded on "green clay ", to avoid imminent collapse of the gable: in Quincy-sous-Senart (Seine-et-Marne).

dont la teneur en eau s'abaisse à $20 \%$ a-t-elle une pression de gonflement de l'ordre de $1 \mathrm{MPa}$ et sa résistance à la compression simple peut passer de 0,2 $\mathrm{MPa}$ pour une teneur en eau de $35 \%$ à plus de 2 $\mathrm{MPa}$ pour une teneur en eau de $20 \%$ (fig. 5).

A partir de 1976, on a revu certaines idées et on a commencé à se préoccuper des problèmes de dessiccation et de retrait ; on a alors estimé à juste titre que les fondations devaient être établies à une profondeur suffisante pour échapper aux phénomènes de variations de teneur en eau. Mais les sécheresses de 1989 et 1990 ont montré que les argiles pouvaient s'assécher sur plusieurs mètres. Un récent sinistre à Paris a ainsi montré que les argiles vertes avaient été desséchées sur $5 \mathrm{~m}$ d'épaisseur. On comprend aisément les désordres qui peuvent en résulter quand une couche d'argile verte de $2 \mathrm{~m}$ d'épaisseur tasse de 4 à $5 \mathrm{~cm}$ lorsque sa teneur en eau s'abaisse de $5 \%$.
C'est pourquoi récemment de nombreux sinistres se sont déclarés sur des constructions qui avaient déjà été reprises en sous-œuvre au moyen de puits manuels dont on pensait qu'ils descendaient assez bas pour échapper aux variations de teneur en eau du sol, mais qui restaient toutefois dans l'argile.

En fait, pour une construction neuve, si l'on veut malgré tout se fonder dans l'argile et éviter de faire des fondations profondes sous la couche argileuse, il faut respecter 4 conditions :

- descendre les fondations aussi bas que possible ; - faire des structures assez raides pour que les mouvements différentiels du sol puissent être absorbés par cette rigidité ;

- éviter toute plantation de type peuplier, saule ou chêne à proximité de la construction (on dit qu'un 


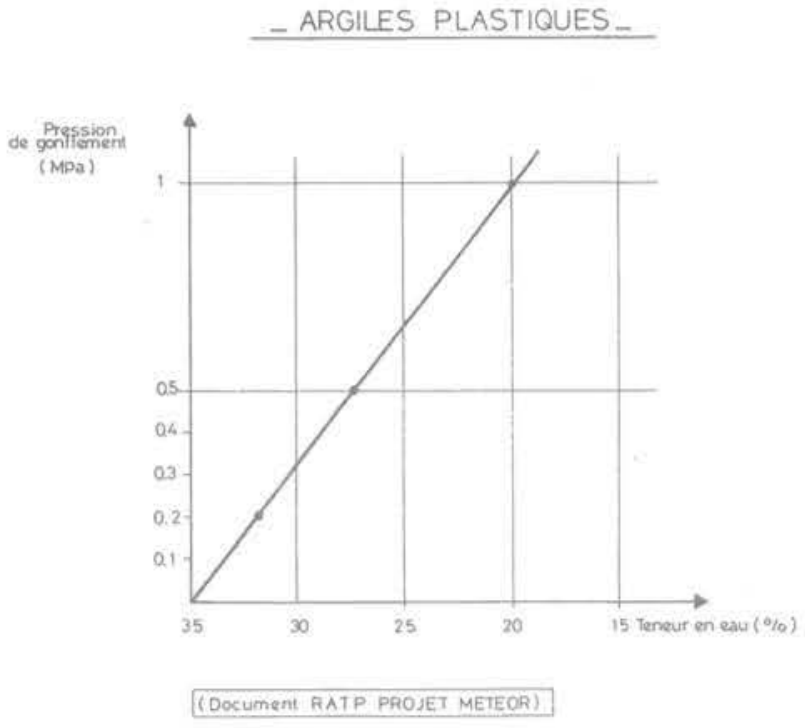

Fig. 5. - Variation de la pression de gonflement en fonction de la teneur en eau.

Fig. 5. - Variation of the swelling pressure us water content.

arbre adulte de l'une de ces espèces dessèche le terrain jusqu'à une distance égale à 1,5 fois sa hauteur) ; - faire des canalisations très résistantes pour éviter tout apport ponctuel d'eau sous les fondations suite à une rupture.

Un cas particulier est constitué par les terrains en pente; il faut veiller à ce que les fondations soient encastrées par rapport au terrain naturel de la même hauteur à l'amont et à l'aval, sinon on s'expose à un assèchement plus intense des argiles à l'aval et donc à un tassement plus accentué à l'aval. Ce phénomène est à l'origine de nombreux sinistres.

\section{ETUDE DE SOLS EN CAS DE SINISTRE}

Dans le cas d'un sinistre dû à un phénomène de retrait d'argile, l'étude de sols aura pour but de déterminer les causes exactes du sinistre et les moyens à mettre en cuvre pour y remédier.

Dans cette optique, il conviendra en premier lieu de déterminer au moyen d'un ou plusieurs puits de reconnaissance la géométrie des fondations, leur profondeur et la nature du terrain d'assise. Cette reconnaissance est indispensable pour concevoir ensuite la reprise en sous-œuvre.

Puis, il faut déterminer la nature et les caractéristiques des terrains sur une hauteur suffisante permettant de dimensionner correctement la reprise en sous-cuvre. A cet égard on doit respecter la prescription du DTU 13.2 « Fondations Profondes » imposant de reconnaître les terrains jusqu'à $5 \mathrm{~m}$ au-dessous des fondations futures. La reconnaissance devra permettre de déterminer :

- le niveau d'assise des puits ou micropieux de reprise en sous-cuvre éventuelle;
- les hypothèses à prendre en compte dans le calcul des forces portantes:

- épaisseur sur laquelle on doit tuber les micropieux ou prendre un frottement négatif :

- frottement latéral positif admissible dans les terrains compacts et insensibles aux variations de teneur en eau ;

- valeur de l'effort admissible par le sol pour des fondations sur puits.

A cet effet, les différentes méthodes qui peuvent être mises en cuvre sont les suivantes:

- le pénétromètre dynamique, mais cet appareil peut donner lieu à de faux refus, d'où une hauteur de reconnaissance insuffisante et par ailleurs, on ne reconnaît pas par cette technique la nature du terrain, et en particulier l'épaisseur d'argile à neutraliser ;

- le pressiomètre, à la condition que les essais pressiométriques soient réalisés dans des sondages à la tarière ou par carottage-battage, de manière à obtenir une coupe précise du terrain ;

- le Standard Pénétration Test. Cette technique qui consiste à carotter le terrain et à mesurer sa résistance est particulièrement bien adaptée, même en terrain argileux, contrairement à un certain nombre d'idées reçues.

Dans les cas difficiles, on complètera ces investigations in situ par des essais en laboratoire : essais de gonflement à l'œdomètre pour apprécier le degré de dessèchement des argiles et leur potentiel de gonflement, mesures de teneur en eau pour déterminer l'épaisseur de terrain qui a été desséchée.

Enfin, il sera utile de suivre l'évolution des désordres et d'apprécier le cas échéant l'efficacité du remède mis en ceuvre. A cet effet, deux systèmes peuvent être utilisés soit ensemble, soit séparément:

- mesures de nivellement direct à partir d'un point supposé fixe (on a ainsi une précision de l'ordre du $1 / 10 \mathrm{~mm}$ ) ;

- mesures d'ouvertures de fissures au moven de jauges à vernier du type LPJS qui donnent aussi une précision du $1 / 10 \mathrm{~mm}$, ou au moyen d'extensomètres à billes qui ont une précision du $1 / 100 \mathrm{~mm}$ et qui permettent des mesures dans les trois dimensions.

Pour être valables, les mesures doivent être effectuées sur un cycle de saisons, c'est-à-dire pendant un an, avec une fréquence si possible mensuelle.

\section{LES DIFFÉRENTS REMÈDES}

Une fois la situation analysée et le diagnostic porté sur l'origine des désordres et sur leur évolution prévisible, il importe de mettre en cuure des moyens propres à assurer la stabilité, si possible définitive, du bâtiment sinistré.

Dans bien des cas, il suffira d'abattre l'arbre (ou les arbres) à l'origine d'une situation dissymétrique. Il faudra non seulement couper l'arbre mais aussi neutraliser définitivement ses racines par injection de chlorate de soude. Il vaut mieux souvent sacrifier quel- 
ques arbres plutôt que de se lancer dans une reprise en sous-cuvre des fondations onéreuse et parfois hasardeuse. Parallèlement à cette opération, il conviendra de vérifier et, le cas échéant, de réparer et de renforcer les canalisations d'eaux pluviales et usées.

Mais parfois, on ne peut pas toujours couper les arbres parce qu'ils sont chez un voisin récalcitrant qui les avait plantés avant que ne soit construit le bâtiment sinistré, ou bien la cause est différente et il faut alors envisager une reprise en sous-œuvre du bâtiment sinistré.

Toutes les reprises en sous-cuvre n'ont pas la même valeur. En premier lieu, il faut éviter, comme on l'a vu, celles qui restent dans les terrains argileux et qui peuvent s'avérer insuffisantes à terme. Il convient également d'éviter les reprises partielles qui entraînent la création de points durs et sont susceptibles d'engendrer des désordres encore pires. Ainsi pour un pavillon en Seine-et-Marne deux angles avaient été repris au moyen de micropieux. L'année suivante il fallut étayer le pavillon qui se disloquait ; il a ensuite été nécessaire de le démolir entièrement et de le reconstruire sur des pieux de gros diamètre (fig. 6).

Ceci étant, la meilleure manière de reprendre un bâtiment en sous-œuvre consiste à réaliser des puits manuels blindés sous les terrains argileux. Ce système présente trois avantages majeurs :

- du point de vue mécanique un puits, massif par nature, a une inertie beaucoup plus forte que les micropieux, ce qui lui permet de mieux résister aux efforts horizontaux qui sont exercés en tête de fondation car les phénomènes de retrait et de gonflement se développent aussi bien dans le sens horizontal que vertical ;

- on peut vérifier la nature et la résistance du terrain que l'on atteint ;

- on peut surtout voir ce que l'on fait alors que dans le cas de micropieux on n'est pas à l'abri d'une erreur d'exécution.

Les puits peuvent être jointifs, auquel cas ils seront exécutés par plots alternés. Ils peuvent aussi être discontinus et liés entre eux par une longrine en béton armé exécutée par parties en sous-œuvre.

Les puits sont coulés en béton non armé dosé à $350 \mathrm{~kg}$ de ciment CLK par $\mathrm{m}^{3}$. La liaison entre les fondations anciennes et les reprises se fait au moyen d'un mortier sans retrait mis en place après que le béton ait fait sa prise. Bien entendu toutes précautions doivent être prises pour éviter d'aggraver les désordres pendant les travaux ; en particulier, on limitera les ouvertures sous les fondations à des plots de 1,5 $\mathrm{m}$ de large (éventuellement davantage en cas de fondations ferraillées).

L'inconvénient majeur de la technique des puits réside dans son prix, si bien que cette technique n'est guère applicable que si la couche portante se trouve à faible profondeur (moins de $5 \mathrm{~m}$ en général).

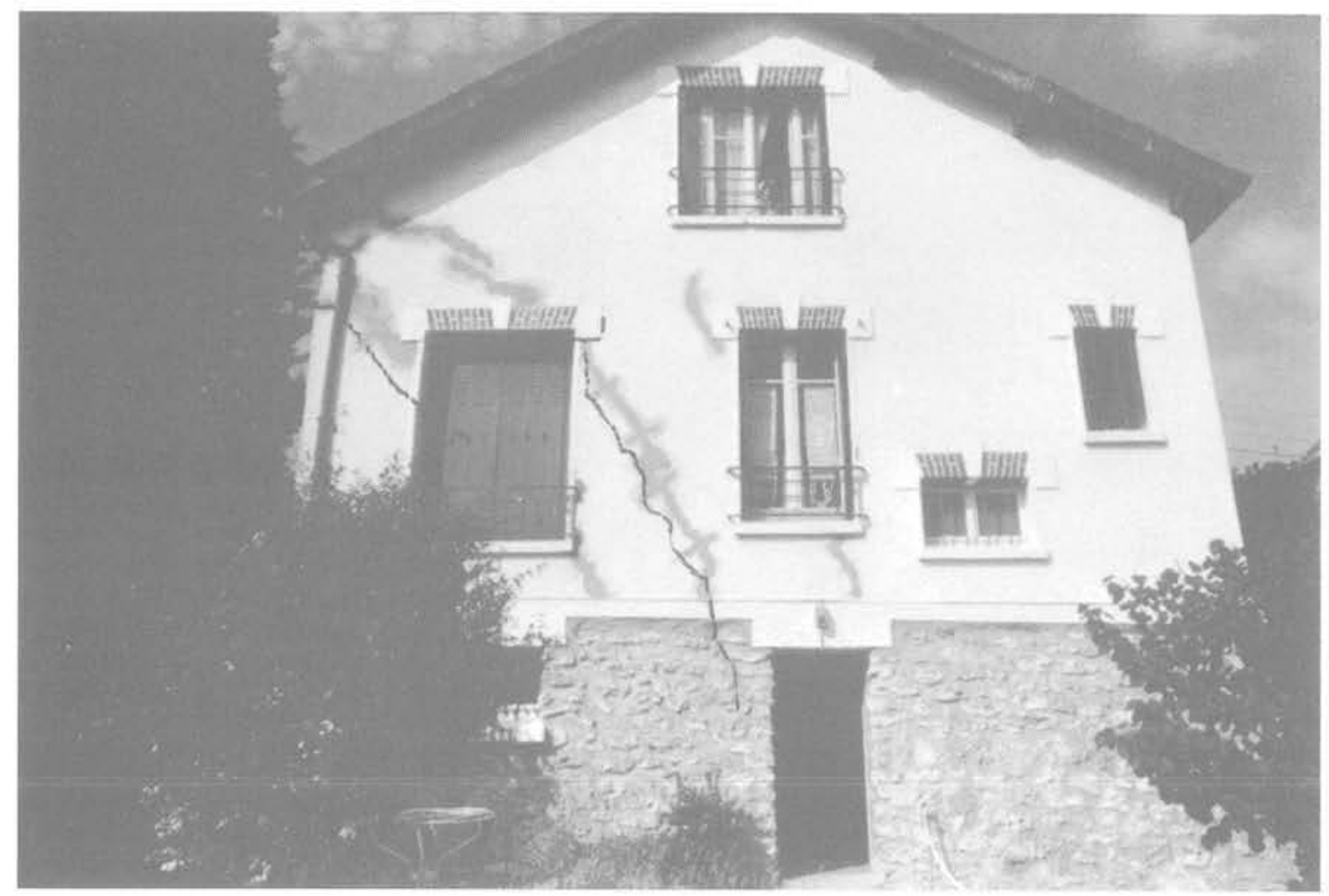

Fig. 6. - (Cliché SIMECSOL). La fissure de l'année 1985 a été colmatée, on a repris en sous-ceuvre le pavillon partiellement au moyen de puits trop courts ... et en 1989 une nouvelle fissure, parallèle à la précédente est apparue. Pavillon situé à Montgeron (Essonne) et fondé sur les argiles vertes.

Fig. 6. - 1985 crack was tapped; the villa was further partially under-piled with too short piles... and in 1989, new crack appeared parallel with the ancient one.

Villa settled in Montgeron (Essonne) and founded on "green clay p. 
La deuxième technique, dont l'emploi se généralise est donc celle des micropieux ancrés largement dans des terrains compacts et insensibles aux variations de teneur en eau. Le liaisonnement aux fondations existantes pourra se faire par scellement direct au mortier sans retrait à la condition que les fondations soient assez épaisses et assez résistantes. Sinon il sera nécessaire de réaliser une longrine de liaison en souscuuvre. A noter que les forages à travers les fondations doivent se faire par carottage pour éviter tout ébranlement des structures d'un bâtiment déjà fragilisé. Les micropieux ont généralement des diamètres de 100 à $200 \mathrm{~mm}$. Ils sont armés de barres type DYWIDAG ou de tubes métalliques dont la section est calculée pour reprendre les charges transmises aux micropieux. Il s'agit de micropieux non injectés où l'espace entre l'armature et le terrain est rempli d'un coulis fortement dosé en ciment $\left(1200 \mathrm{~kg} / \mathrm{m}^{3}\right)$ mis en place par gravité au tube plongeur.

Le calcul des micropieux devra tenir compte du fait que lorsque les terrains se réhydratent des forces ascendantes dues au gonflement peuvent se développer si bien que les micropieux travaillent alors en tirants. Ainsi s'ils ne sont pas assez ancrés dans la couche compacte sous l'argile, ils peuvent être entraînés vers le haut. On a vu ainsi dans le Val-de-Marne des maisons reprises par des micropieux trop courts, se soulever, et subir de tels désordres qu'il a fallu les démolir et les reconstruire (en réalisant là encore des fondations sur pieux de gros diamètre).

Dans tous les cas, les réparations ne devront être entreprises qu'après stabilisation des mouvements. En effet, les travaux de reprises en sous-œuvre peuvent engendrer de nouveaux mouvements et surtout, il convient d'attendre que les charges soient effectivement reportées sur les fondations nouvelles; or ces fondations n'entreront véritablement en service qu'à l'été suivant lorsque les fondations anciennes connaîtront à nouveau une défaillance sous l'effet de la sécheresse.

Enfin, il convient de parler de deux techniques pour les déconseiller, sauf dans certains cas bien précis. En premier lieu, le drainage qui contribue encore à assé- cher le terrain, donc à accentuer le phénomène de retrait. En second lieu l'arrosage du terrain qui ne peut être efficace que s'il est fait de manière uniforme, régulière et dosée, autant de conditions dont on s'aperçoit qu'elles sont difficiles à réunir dans la pratique.

\section{LA CONDUITE DES TRAVAUX DE CONFORTEMENT}

La conduite des travaux de confortement nécessite beaucoup de soin. Il faut d'abord porter le bon diagnostic, puis établir un projet qui soit bien adapté au problème posé, car chaque cas est particulier, et enfin s'assurer de la bonne exécution des travaux et de leur efficacité. C'est pourquoi il est indispensable que la direction de ces travaux soit confiée à un maître d'œuvre spécialisé qui conseillera le maître d'ouvrage et défendra au mieux ses intérêts technique et financier.

C'est ce maître d'œuvre qui établira le projet sur la base duquel seront consultées les entreprises, c'est lui qui vérifiera que les conditions d'exécution sont correctes et qui sera à même de règler les problèmes en cours de chantier. Le maître d'œuvre pourra être le géotechnicien qui a procédé aux études de sols à la condition bien entendu qu'il ait les qualifications voulues et qu'il soit assuré en conséquence.

La présence d'un bureau de contrôle est également souhaitable bien que non indispensable du moins pour les pavillons individuels. Enfin, il convient que le maître d'ouvrage souscrive une assurance dommagesouvrages. Il $y$ a en effet un très grand nombre de sinistres dus à des reprises insuffisantes ou mal exécutées.

Les phénomènes naturels dus au dessèchement des argiles mettent en jeu des forces considérables. Il reste encore beaucoup à observer et à apprendre dans ce domaine, qu'il convient d'aborder avec sérieux et modestie. 